Algebraic 83 Geometric $\mathcal{T}$ opology

Volume 4 (2004) 813-827

Published: 23 September 2004

ATG

\title{
On the homotopy invariance of configuration spaces
}

\author{
Mokhtar Aouina \\ JOHN R. KLEIN
}

\begin{abstract}
For a closed PL manifold $M$, we consider the configuration space $F(M, k)$ of ordered $k$-tuples of distinct points in $M$. We show that a suitable iterated suspension of $F(M, k)$ is a homotopy invariant of $M$. The number of suspensions we require depends on three parameters: the number of points $k$, the dimension of $M$ and the connectivity of $M$. Our proof uses a mixture of Poincaré embedding theory and fiberwise algebraic topology.
\end{abstract}

AMS Classification 55R80; 57Q35, 55R70

Keywords Configuration space, fiberwise suspension, embedding up to homotopy, Poincaré embedding

\section{Introduction}

For a closed PL manifold $M$ and an integer $k \geq 2$, we will consider the configuration space

$$
F(M, k):=\left\{\left(x_{1}, \ldots, x_{k}\right) \mid x_{i} \in M \text { and } x_{i} \neq x_{j} \text { for } i \neq j\right\} .
$$

A fundamental unsolved problem about these spaces concerns their homotopy invariance: when $M$ and $N$ are homotopy equivalent, is it true that $F(M, k)$ and $F(N, k)$ are homotopy equivalent?

Here is some background. It is known that the based loop space $\Omega F(M, k)$, is a homotopy invariant (see Levitt $[\mathrm{L}$ ). When $M$ is smooth, the cohomology of $F(M, k)$ with field coefficients has been intensively studied (see e.g., Bödigheimer-Cohen-Taylor [B-C-T] $)$. When $M$ is a smooth projective variety over $\mathbb{C}$, Kriz $[\mathrm{Kr}]$ has shown that the rational homotopy type of $F(M, k)$ depends only on the rational cohomology ring of $M$.

When $k=2$ we have $F(M, 2)=M \times M-\Delta$ is the deleted product. Even in this instance, the homotopy invariance question is still not completely settled. 
However, shortly after the first draft of this paper was circulated, R. Longoni and P. Salvatore partially settled the question by showing that the deleted product spaces of the homotopy equivalent (but not simple homotopy equivalent) lens spaces $L(7,1)$ and $L(7,2)$ have distinct homotopy types (see [L-S]).

The purpose of this paper is to show that a suitable iterated suspension of $F(M, k)$ is a homotopy invariant. The bound on the number of suspensions we need to take depends on three parameters: the number of points, the dimension of $M$ and the connectivity of $M$.

For an unbased space $Y$, we define its $j$-fold (unreduced) suspension

$$
\Sigma^{j} Y:=\left(* \times S^{j-1}\right) \cup\left(Y \times D^{j}\right),
$$

where the union is amalgamated along $Y \times S^{j-1}$ (up to homotopy, $\Sigma^{j} Y$ is the join of $Y$ and $\left.S^{j-1}\right)$.

Our main result is

Theorem A Let $M$ and $N$ be homotopy equivalent closed PL manifolds of dimension $d$. Assume $M$ is $r$-connected for some $r \geq 0$. Then there is a homotopy equivalence

$$
\Sigma^{\alpha(k, d, r)} F(M, k) \simeq \Sigma^{\alpha(k, d, r)} F(N, k),
$$

where $\alpha(k, d, r):=\max ((k-2) d-r+3,0)$.

Remark Cohen and Taylor (unpublished manuscript) prove by very different methods that the configuration spaces of smooth manifolds are stable homotopy invariant. In their work the bound on the number suspensions required to achieve homotopy invariance is significantly weaker.

Nevertheless, an advantage of their approach is its applicability to other kinds of configuration spaces. For example, their results apply as well to the unordered configuration spaces of a smooth manifold. We are unable to analyze the latter using our methods.

Corollary B Let $M$ be a connected closed PL manifold. Then the suspension spectrum $\Sigma^{\infty} F(M, k)_{+}$is a homotopy invariant of $M$.

Theorem $\mathrm{A}$ can be improved by one dimension provided that the input manifolds are simple homotopy equivalent: 
Theorem C With the additional assumption that $M$ and $N$ are simple homotopy equivalent, there is a homotopy equivalence

$$
\Sigma^{\beta(k, d, r)} F(M, k) \simeq \Sigma^{\beta(k, d, r)} F(N, k),
$$

where $\beta(k, d, r):=\max ((k-2) d-r+2,0)$.

Remark For simply connected manifolds, a homotopy equivalence is also simple. Thus Theorem $[$ improves upon Theorem $\mathrm{A}$ in the 1-connected case.

The following corollary extends the work of Levitt $[\mathrm{L}$, who considered only the case of 2-connected manifolds.

Corollary D If $M$ is connected, then $\Sigma^{3} F(M, 2)$ is a homotopy invariant of $M$. Furthermore, if $M$ is 1 -connected, then $\Sigma F(M, 2)$ is a homotopy invariant of $M$.

(The first part is just a special case of Theorem $\mathrm{A}$ whereas the second part is a special case of Theorem C)

Conventions We work in the category Top of compactly generated topological spaces.

A non-empty space is always $(-1)$-connected. A space is 0 -connected if it is path connected. It is $r$-connected for $r>0$ if it is path connected and its homotopy groups (with respect to a choice of basepoint) vanish in degrees $\leq r$. A map $A \rightarrow B$ of spaces is $r$-connected if its homotopy fiber at all basepoints is $(r-1)$-connected. A weak (homotopy) equivalence is an $\infty$-connected map. If two spaces $A$ and $B$ are related by a chain of weak equivalences, we will often indicate it by writing $A \simeq B$. A space is homotopy finite if it is homotopy equivalent to a finite cell complex.

Outline In $\S 2$ we describe the construction of fiberwise suspension and deduce some elementary properties of it. In $\S 3$ we review the Stallings-Wall theory of embeddings up to homotopy. $\S 4$ is about decompressing embeddings up to homotopy so as to increase their codimensions. A key result of this section concerns the iterated suspension of the complement of an embedding up to homotopy. In $\S 5$ Theorem $[$ is proved using the Browder-Casson-Sullivan-Wall theorem and the Stallings-Wall embedding theorem. In $\S 6$ Theorem $\mathrm{A}$ is proved using the second author's previous work on Poincaré embeddings. 
Acknowledgements We are indebted to the referee for pointing out a mistake we made when applying the Browder-Casson-Sullivan-Wall theorem in a previous version of this paper. The mistake evaporates when one assumes simple homotopy equivalences between the input manifolds: Theorem [C] is an artifact of our original (erroneous) proof of Theorem A

The proof of Theorem $\mathrm{A}$ contained here uses a result of the second author on concordances between Poincaré embeddings; the latter accounts for the loss of one dimension in the statement of Theorem $\mathrm{A}$

The first author is supported by a Wayne State University Rumble Fellowship. The second author is partially supported by NSF Grant DMS-0201695.

\section{Fiberwise suspension}

Let $A \rightarrow X$ be a map of spaces. Define

$$
\operatorname{Top}_{A \rightarrow X}
$$

to be the category of spaces "between $A$ and $X$." Specifically, an object is a space $Y$ and a choice of factorization $A \rightarrow Y \rightarrow X$. A morphism is a map of spaces which is compatible with their given factorizations. Call a morphism a weak equivalence if it is a weak homotopy equivalence of underlying spaces.

We write $\boldsymbol{T o p}_{/ X}$ for $\boldsymbol{T o p}_{\emptyset \rightarrow X}$. If $Y \in \mathbf{T o p}_{/ X}$ is an object, define its (unreduced) $j$-fold fiberwise suspension by

$$
\Sigma_{X}^{j} Y:=\left(Y \times D^{j}\right) \cup\left(X \times S^{j-1}\right),
$$

where the union is amalgamated over $Y \times S^{j-1}$. With respect to the first factor projection map $X \times S^{j-1} \rightarrow X$, we get a functor

$$
\Sigma_{X}^{j}: \operatorname{Top}_{/ X} \rightarrow \operatorname{Top}_{X \times S^{j-1} \rightarrow X} .
$$

Lemma 2.1 Let $Y$ and $Z$ be objects of Top $/ X$ whose underlying spaces are path connected and have the homotopy type of $C W$ complexes.

Assume for some $j \geq 0$ that $\Sigma_{X}^{j} Y$ and $\Sigma_{X}^{j} Z$ are weak equivalent objects. Then there is a weak equivalence of spaces

$$
\Sigma^{j} Y \simeq \Sigma^{j} Z .
$$


Proof The statement is obviously true for $j=0$, so we will assume that $j>0$. Moreover, we may assume that we are given a weak equivalence $\Sigma_{X}^{j} Y \stackrel{\sim}{\rightarrow} \Sigma_{X}^{j} Z$. For any object $T \in \mathbf{T o p}_{/ X}$, we have a cofibration sequence of spaces

$$
X \times S^{j-1} \rightarrow \Sigma_{X}^{j} T \rightarrow \Sigma^{j}\left(T_{+}\right),
$$

where we use that $\Sigma^{j}\left(T_{+}\right)$is $T \times D^{j}$ with $T \times S^{j-1}$ collapsed to a point. Using this cofiber sequence for both $Y$ and $Z$, we get a commutative diagram

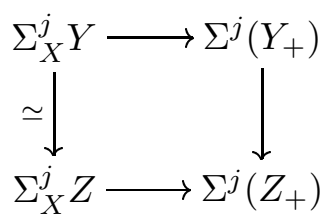

which is also homotopy pushout. It is well-known that cobase change preserves weak equivalences (see e.g., Hirschhorn $[\mathrm{H}]$ ), so it follows that the map $\Sigma^{j}\left(Y_{+}\right) \rightarrow \Sigma^{j}\left(Z_{+}\right)$is a weak equivalence.

If $A$ is a space, let $A_{+}$be the union of $A$ with a disjoint basepoint. Then one has a weak equivalence

$$
\Sigma^{j}\left(A_{+}\right) \simeq\left(\Sigma^{j} A\right) \vee S^{j},
$$

for $j>0$, where the left side is to be regarded as the reduced suspension of a based space.

An explicit weak equivalence can be constructed as follows: recall that

$$
\Sigma^{j} A=\left(A \times D^{j}\right) \cup_{A \times S^{j-1}}\left(* \times S^{j-1}\right) .
$$

The effect of collapsing $* \times S^{j-1}$ to a point defines a based map

$$
i: \Sigma^{j} A \rightarrow \Sigma^{j}\left(A_{+}\right) \text {. }
$$

Choosing a basepoint for $A$ yields a based map $S^{0} \rightarrow A_{+}$. Let $k: S^{j} \rightarrow \Sigma^{j}\left(A_{+}\right)$ denote its $j$-fold suspension. Then we obtain a map

$$
\left(S^{j} A\right) \vee S^{j} \stackrel{i \vee k}{\longrightarrow} \Sigma^{j}\left(A_{+}\right) \vee \Sigma^{j}\left(A_{+}\right) \stackrel{\text { fold }}{\longrightarrow} \Sigma^{j}\left(A_{+}\right) .
$$

It is straightforward to check that this map is a weak equivalence.

Consequently, we have weak equivalences $\Sigma^{j}\left(Y_{+}\right) \simeq\left(\Sigma^{j} Y\right) \vee S^{j}$ and $\Sigma^{j}\left(Z_{+}\right) \simeq$ $\left(\Sigma^{j} Z\right) \vee S^{j}$ for $j>0$. It follows that there is a weak equivalence

$$
\left(\Sigma^{j} Y\right) \vee S^{j} \simeq\left(\Sigma^{j} Z\right) \vee S^{j} .
$$

Because $Y$ and $Z$ are connected, we have that $\Sigma^{j} Y$ and $\Sigma^{j} Z$ are $j$-connected. Using Lemma 2.2 below, we conclude that the composite

$$
\Sigma^{j} Y \stackrel{\text { include }}{\longrightarrow}\left(\Sigma^{j} Y\right) \vee S^{j} \simeq\left(\Sigma^{j} Z\right) \vee S^{j} \stackrel{\text { project }}{\longrightarrow}\left(\Sigma^{j} Z\right)
$$

is a weak equivalence. 
Lemma 2.2 Let $U$ and $V$ be $j$-connected spaces with $j \geq 0$. Assume $U$ and $V$ are equipped with non-degenerate basepoints. Assume $h: U \vee S^{j} \rightarrow V \vee S^{j}$ is a weak equivalence. Then the composition

$$
g: U \stackrel{\text { include }}{\longrightarrow} U \vee S^{j} \stackrel{h}{\longrightarrow} V \vee S^{j} \stackrel{\text { project }}{\longrightarrow} V
$$

is also a weak equivalence.

Proof Without loss in generality we can assume that $U$ and $V$ are CW complexes with no cells in positive dimensions $\leq j$. By cellular approximation, we may also assume that $h$ is a cellular map. Then $h$ preserves $j$-skeleta, so there is a commutative diagram

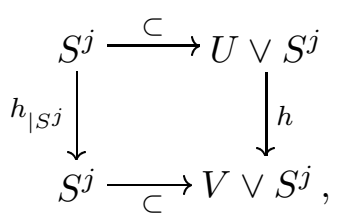

and it is straightforward to check that the left vertical map is a homotopy equivalence. We infer that the map $U \rightarrow V$ obtained by taking cofibers horizontally is also a weak equivalence. But this map coincides with $g$.

\section{Embeddings up to homotopy}

Let $K$ be a space which is homeomorphic to a connected finite complex of dimension $\leq k$. Let $M$ be a PL manifold of dimension $d$, possibly with boundary. Fix a map $f: K \rightarrow M$.

Definition 3.1 An embedding up to homotopy of $f$ is a pair

$$
(N, h)
$$

in which

- $N$ denotes a compact codimension zero PL submanifold of the interior of $M$;

- the pair $(N, \partial N)$ is $(n-k-1)$-connected;

- $h: K \rightarrow N$ is a simple homotopy equivalence such that composition

$$
K \stackrel{h}{\rightarrow} N \subset M
$$

is homotopic to $f$. 
A concordance of embeddings up to homotopy $\left(N_{0}, h_{0}\right)$ and $\left(N_{1}, h_{1}\right)$ of $f$ consists of a locally flat embedding

$$
e: N_{0} \times[0,1] \subset M \times[0,1]
$$

and a homotopy $H_{t}: K \rightarrow N_{0}$ such that

- $e$ restricted to $N_{0} \times 0$ is the inclusion and $e$ maps $N_{0} \times 1$ homeomorphically onto $N_{1}$.

- $H_{0}=h_{0}$ and $H_{1}$ followed by $e(\cdot, 1)$ coincides with $h_{1}$.

Theorem 3.2 (Stallings [St, Wall Wa1]) Assume $\operatorname{dim} K \leq k \leq d-3$. If $f: K \rightarrow M$ is $(2 k-d+1)$-connected, then $f$ embeds up to homotopy. Furthermore, any two embeddings up to homotopy of $f$ are concordant whenever $f$ is $(2 k-d+2)$-connected.

\section{Decompression}

Let $(N, h)$ be an embedding up to homotopy of $f: K \rightarrow M$. If $C$ denotes the closure of the complement of $N$ inside $M$, then $C$ is an object of $\mathbf{T o p}_{\partial M \subset M}$.

Definition 4.1 The object

$$
C \in \mathbf{T o p}_{\partial M \subset M}
$$

is called the complement of $(N, h)$.

By considering the inclusion $M \times 0 \subset M \times D^{j}$, and taking a compact regular neighborhood of $N$ in $M \times D^{j}$, we have an associated embedding up to homotopy of the composite

$$
f_{j}: K \stackrel{f}{\rightarrow} M=M \times 0 \subset M \times D^{j} .
$$

Denote this embedding up to homotopy by $\left(N_{j}, h_{j}\right)$, where $N_{j} \cong N \times D_{1 / 2}^{j}$ (here $D_{1 / 2}^{j} \subset D^{j}$ is the disk of radius $1 / 2$ ) and $h_{j}$ is identified with $h$ followed

by the inclusion $N \times 0 \subset N \times D_{1 / 2}^{j}$. This new embedding up to homotopy is the $j$-fold decompression of $(N, h)$. Note that its complement has the structure of an object of $\operatorname{Top}_{\partial\left(M \times D^{j}\right) \subset M \times D^{j}}$.

However, to avoid technical problems, we will henceforth regard the complement as a space over $M$ by projecting away from the $D^{j}$ factor. That is, we will think of the complement as an object of $\mathbf{T o p}_{\partial\left(M \times D^{j}\right) \rightarrow M}$. 
Lemma 4.2 (Compare [Kl, §2.3]) Assume that $M$ is closed. Then the complement of $\left(N_{j}, h_{j}\right)$ is weak equivalent to the object

$$
\Sigma_{M}^{j} C
$$

Proof The regular neighborhood $N_{j}$ can be chosen as $N \times D_{1 / 2}^{j} \subset M \times D^{j}$. The complement of $\left(N_{j}, h_{j}\right)$ is then

$$
\left(M \times D^{j}\right)-\operatorname{int}\left(N \times D_{1 / 2}^{j}\right)=C \times D_{1 / 2}^{j} \cup M \times D_{[1 / 2,1]}^{j},
$$

where $D_{[1 / 2,1]}^{j}$ denotes the annulus consisting of points in $D^{j}$ whose norm varies between $1 / 2$ and 1 . The above union is amalgamated over $C \times \partial D_{1 / 2}^{j}$.

The subspace of the complement given by $\left(C \times D_{1 / 2}^{j}\right) \cup\left(M \times \partial D_{1 / 2}^{j}\right)$ is evidently isomorphic to $\Sigma_{M}^{j} C$. The inclusion map of this subspace is, up to isomorphism, a morphism of $\mathbf{T o p}_{M \times S^{j-1} \rightarrow M}$. Furthermore, this inclusion is a weak homotopy equivalence of the underlying spaces.

We conclude this section with a result about the homotopy type of the iterated suspension of the complements of embeddings up to homotopy. This will be a key ingredient of the proof of Theorem $\mathrm{C}$

Proposition 4.3 Assume $f: K^{k} \rightarrow M^{d}$ is an $r$-connected map, where $M$ is a closed connected PL manifold of dimension $d$, and $\operatorname{dim} K \leq k \leq d-3$. Suppose that $f$ has two embeddings up to homotopy $(N, h)$ and $\left(N^{\prime}, h^{\prime}\right)$ with respective complements $C$ and $C^{\prime}$. Then there is a homotopy equivalence,

$$
\Sigma^{j} C \simeq \Sigma^{j} C^{\prime}
$$

where $j=\max (2 k-d-r+2,0)$.

Proof By the Stallings-Wall theorem (3.2), with $j=\max (2 k-d-r+2,0)$, we see that the $j$-fold decompressions of $(N, h)$ and $\left(N^{\prime}, h^{\prime}\right)$ are concordant. Furthermore, it is evident from the definitions that concordant embeddings up to homotopy have homotopy equivalent complements.

Using Lemma 4.2 we infer that there is a weak equivalence of objects

$$
\Sigma_{M}^{j} C \simeq \Sigma_{M}^{j} C^{\prime}
$$

By Lemma 2.1, we conclude $\Sigma^{j} C \simeq \Sigma^{j} C^{\prime}$. 


\section{Proof of Theorem C}

Suppose that $M$ and $N$ are simple homotopy equivalent $r$-connected $(r \geq 0)$ closed PL manifolds of dimension $d$.

With appropriate modifications, we will argue along the lines of Levitt's strategy for showing $F(M, 2) \simeq F(N, 2)$ when $M$ and $N$ are 2-connected (see $[\mathrm{L}$ ).

\section{Case $1 \quad d \leq 2$}

By the classification of low dimensional manifolds, $M$ and $N$ are PL homeomorphic. It follows that $F(M, k)$ and $F(N, k)$ are homeomorphic for all $k$.

\section{Case $2 d>2$}

Let

$$
\Delta_{k}^{\mathrm{fat}}(M) \subset M^{\times k}
$$

denote the fat diagonal. This subpolyhedron is the space of $k$-tuples of points of $M$ such that at least two entries in the $k$-tuple coincide.

By choosing a compact regular neighborhood $V \subset M^{\times k}$ of the fat diagonal, we obtain an embedding up to homotopy of the inclusion $\Delta_{k}^{\mathrm{fat}}(M) \subset M^{\times k}$. Its complement $C$ is weak equivalent to $F(M, k)$ when the latter is considered as an object of Top $_{/ M \times k}$. Denote this embedding up to homotopy by $(V, h)$.

Then we obtain a manifold triad

$$
\left(M^{\times k} ; V, C ; \partial V\right)
$$

(this notation means that $M^{\times k}$ is expressed as a union of the submanifolds $V$ and $C$, with $V \cap C=\partial V=\partial C$ ).

Repeat this procedure for the fat diagonal of $N$ in $N^{\times k}$ to get an embedding up to homotopy of the inclusion $\Delta_{k}^{\text {fat }}(N) \subset N^{\times k}$. Call the latter embedding up to homotopy $\left(W, h^{\prime}\right)$. Its complement $D$ is identified with $F(N, k) \in \operatorname{Top}_{/ N \times k}$. Thus we have another manifold triad

$$
\left(N^{\times k} ; W, D ; \partial W\right) \text {. }
$$

The next step is to choose a simple homotopy equivalence $g: N \stackrel{\sim}{\rightarrow} M$. The $k$-fold product of $g$ with itself produces simple homotopy equivalence of pairs

$$
g_{k}:\left(N^{\times k}, \Delta_{k}^{\mathrm{fat}}(N)\right) \stackrel{\sim}{\rightarrow}\left(M^{\times k}, \Delta_{k}^{\mathrm{fat}}(M)\right) .
$$


Using the Browder-Casson-Sullivan-Wall theorem [Wa2, Th. 12.1] applied to $g_{k}: N^{\times k} \rightarrow M^{\times k}$ and the triad $\left(M^{\times k} ; V, C ; \partial V\right)$, there exists another manifold triad decomposition of $N^{\times k}$, say

$$
\left(N^{\times k} ; V^{\prime}, C^{\prime} ; \partial V^{\prime}\right)
$$

and a simple homotopy equivalence of triads

$$
\phi:\left(N^{\times k} ; V^{\prime}, C^{\prime} ; \partial V^{\prime}\right) \stackrel{\sim}{\rightarrow}\left(M^{\times k} ; V, C ; \partial V\right)
$$

such that $\phi: N^{\times k} \rightarrow M^{\times k}$ is homotopic to $g_{k}$. These data describe another embedding up to homotopy of the inclusion $\Delta_{k}^{\text {fat }}(N) \rightarrow N^{\times k}$ with the property that its complement $C^{\prime}$ is identified with $F(M, k)$ up to homotopy equivalence.

Summarizing thus far, we have two embeddings up to homotopy of the inclusion $\Delta_{k}^{\text {fat }}(N) \rightarrow N^{\times k}$, one whose complement is identified with $F(N, k)$ and the other whose complement is identified with $F(M, k)$.

The next step of the argument is to verify the hypotheses of Proposition 4.3. One checks by elementary means that $\operatorname{dim} \Delta_{k}^{\mathrm{fat}}(N) \leq(k-1) d$. As $d>2$, the hypothesis $(k-1) d \leq k d-3$ is satisfied. Furthermore, the inclusion map $\Delta_{k}^{\mathrm{fat}}(N) \rightarrow N^{\times k}$ is $r$-connected (recall that $r$ is the connectivity of $N$ ). Hence, applying 4.3 we infer

$$
\Sigma^{j} D \simeq \Sigma^{j} C^{\prime}
$$

where $j=\max (2(k-1) d-k d-r+2,0)$. This is precisely the case when $j=\max ((k-2) d-r+2,0)=\beta(k, d, r)$.

Finally, recall that $D \simeq F(N, k)$ and $C^{\prime} \simeq F(M, k)$. With respect to these identifications, we get

$$
\Sigma^{j} F(M, k) \simeq \Sigma^{j} F(N, k) .
$$

This concludes the proof of Theorem [C]

\section{Poincaré embeddings and the proof of Theorem $\mathrm{A}$}

The proof of Theorem $\mathrm{A}$ will use the second author's work on Poincaré embeddings from [Kl] and [Kl2]. The material of this section is not intended to be complete. For the foundations of the theory of Poincaré spaces, see Wa3] and Kl3. 


\section{Motivation}

Let $M$ be a manifold. If $K \subset M$ is a compact codimension zero submanifold, then we get a stratification of $M$ by submanifolds in which the codimension zero stratum consists of $K \amalg \operatorname{cl}(M-K)$ and the codimension one stratum is $\partial K$.

The notion of Poincare embedding is a generalization of this with the manifolds replaced by Poincaré spaces and the stratification replaced by "stratification up to homotopy:"

Definition 6.1 (cf. Kl] ). Let $K$ be a space which is homotopy equivalent to a finite complex of dimension $k$, let $(M, \partial M)$ a Poincaré duality space of dimension $m$ and let $f: K \rightarrow M$ be a map. A (Poincaré) embedding* of $f$ consists of a commutative diagram of homotopy finite spaces

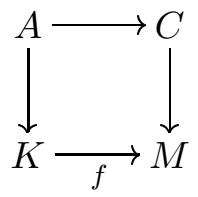

and a factorization $\partial M \rightarrow C \rightarrow M$ such that

- the diagram is homotopy cocartesian, i.e., the map

$$
K \times 0 \cup A \times[0,1] \cup C \times 1 \rightarrow M
$$

is a weak homotopy equivalence;

- if $\bar{K}$ is the mapping cylinder of $A \rightarrow K$, then $\bar{K}$ is an $m$-dimensional Poincaré space with boundary $A$;

- if $\bar{C}$ is the mapping cylinder of $A \amalg \partial M \rightarrow C$, then $\bar{C}$ is an $m$-dimensional Poincaré space with boundary $A \amalg \partial M$;

- there are (compatible) fundamental classes for $\bar{K}$ and $\bar{C}$ which glue to give a fundamental class for $M$;

- the map $A \rightarrow K$ is $(m-k-1)$-connected.

The object $C \in \mathbf{T o p}_{\partial M \subset M}$ is called the complement of the (Poincaré) embedding.

We next turn to the definition of concordance. Roughly, a concordance can be envisioned as a "stratified h-cobordism" between two Poincaré embeddings.

\footnotetext{
*The terminology used here differs slightly from the second author's other papers.
} 
Definition 6.2 (cf. $\left[\mathrm{Kl2}\right.$ ) Assume we are given maps $f_{i}: K \rightarrow M$ for $i=0,1$ which come equipped with embeddings having associated diagram

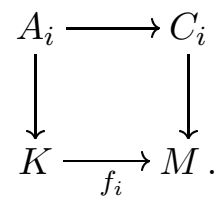

A concordance consists of an extension of these data to a homotopy

$$
F: K \times[0,1] \rightarrow M \times[0,1]
$$

from $f_{0}$ to $f_{1}$, and a commutative diagram of homotopy finite pairs

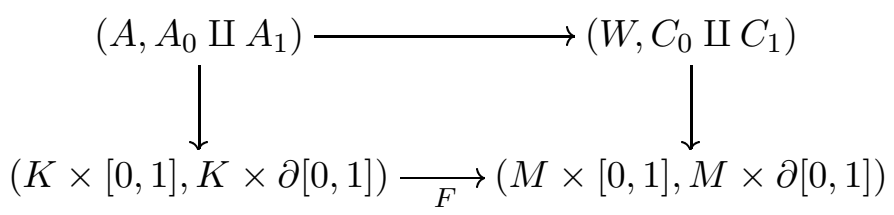

together with a factorization

$$
((\partial M) \times[0,1], \partial M \times \partial[0,1]) \rightarrow\left(W, C_{0} \amalg C_{1}\right) \rightarrow(M \times[0,1], M \times \partial[0,1])
$$

such that

- the diagram is homotopy cocartesian;

- the inclusions $A_{i} \rightarrow A$ and $C_{i} \rightarrow W$ are homotopy equivalences.

Relevant to the proof Theorem $\mathrm{A}$ is the following immediate consequence of the definition: a concordance produces a space $W$ and a commutative diagram

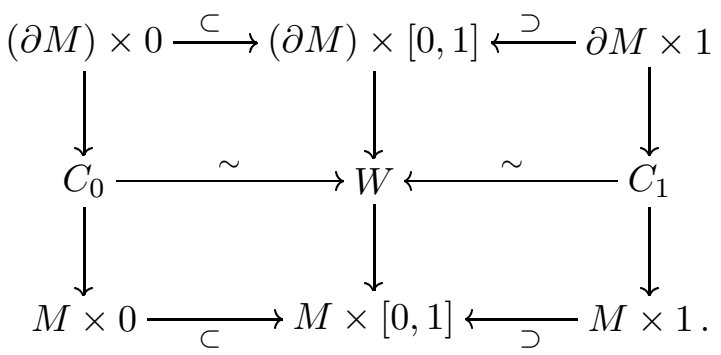

where the horizontal arrows are homotopy equivalences. In particular, the complements of concordant embeddings are weak equivalent objects of $\mathbf{T o p}_{/ M}$.

The following is the key result used in the proof of Theorem A Note the loss of one dimension when compared with the manifold case. 
Theorem 6.3 ([K12, Cor. B]) Let $f_{i}: K \rightarrow M$ for $i=0,1$ be homotopic maps. Assume that $f_{i}$ come equipped with (Poincaré) embeddings. In addition, assume $k \leq m-3$.

If $f_{0}: K \rightarrow M$ is $(2 k-m+3)$-connected, then the embeddings are concordant. In particular, the embeddings have weak equivalent complements.

\section{Decompression}

Given a Poincaré embedding diagram

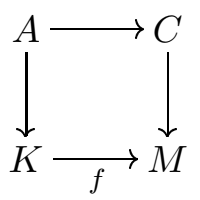

together with factorization $\partial M \rightarrow C \rightarrow M$, apply fiberwise suspension to obtain a new embedding diagram

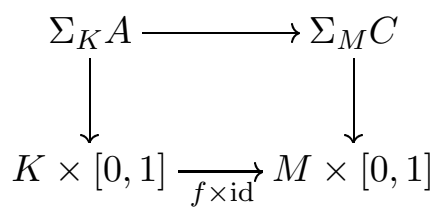

together with factorization $\partial(M \times[0,1])=\Sigma_{M} \partial M \rightarrow \Sigma_{M} C \rightarrow M \times[0,1]$. This operation is called decompression.

If we identify $K \times[0,1]$ with $K$ via first factor projection, we see that decompression increases the codimension (i.e., $m-k$ ) of the original embedding by one. If we iterate the procedure sufficiently many times, we eventually get into the range where Theorem 6.3 applies.

Hence, using Theorem 6.3 together with Lemma 2.1] we infer

Corollary 6.4 Let $f_{i}: K \rightarrow M$ for $i=0,1$ be homotopic maps. Assume that $f_{i}$ come equipped with Poincaré embeddings with complements $C_{i} \rightarrow M$. In addition, assume $f_{i}$ is $r$-connected and $k \leq m-3$.

Then there is a homotopy equivalence of spaces

$$
\Sigma^{j} C_{1} \simeq \Sigma^{j} C_{2}
$$

where $j=\max (2 k-m-r+3,0)$. 
Proof of Theorem \$ Let $h: M \rightarrow N$ be a homotopy equivalence of $r$ connected closed PL manifolds of dimension $d$. If $d \leq 2$, then $M$ and $N$ are homeomorphic and the result is trivial. From now on, assume $d>2$. Let $h_{k}: M^{\times k} \rightarrow N^{\times k}$ be the $k$-fold cartesian product of $h$ with itself.

Using the same notation as in the proof of Theorem Cl we have manifold triads $\left(M^{\times k} ; V, C ; \partial V\right)$ and $\left(N^{\times k} ; W, D ; \partial W\right)$, where $V$ is a regular neighborhood of the fat diagonal $\Delta_{k}^{\mathrm{fat}}(M), W$ is a regular neighborhood of $\Delta_{k}^{\mathrm{fat}}(N), C$ is identified with the configuration space $F(M, k)$ and $D$ with the configuration space $F(N, k)$.

Then, using the identification $V \simeq \Delta_{k}^{\mathrm{fat}}(M)$, the first triad can be regarded as an embedding diagram

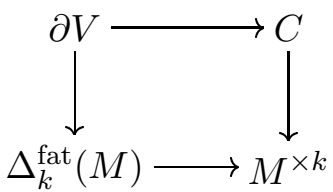

and a similar remark applies to the other triad, to give an embedding diagram

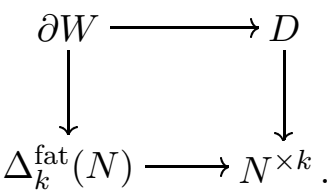

Applying the homotopy equivalence of pairs

$$
h_{k}:\left(M^{\times k}, \Delta_{k}^{\mathrm{fat}}(M)\right) \stackrel{\sim}{\rightarrow}\left(N^{\times k}, \Delta_{k}^{\mathrm{fat}}(N)\right),
$$

to the bottom of the first diagram, we obtain another embedding diagram

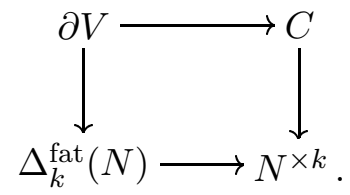

Thus far, what we have achieved is two Poincaré embeddings of the inclusion $\Delta_{k}^{\text {fat }}(N) \rightarrow N^{\times k}$, one having complement $C \rightarrow M^{\times k} \rightarrow N^{\times k}$ and the other with complement $D \rightarrow N^{\times k}$.

Applying Corollary 6.4, we see that

$$
\Sigma^{\alpha(k, d, r)} C \simeq \Sigma^{\alpha(k, d, r)} D .
$$

The proof is now concluded by referring back to the identifications $C \simeq F(M, k)$ and $D \simeq F(N, k)$. 


\section{References}

[B-C-T] Bödigheimer, C.-F., Cohen, F., Taylor, L.: On the homology of configuration spaces. Topology 28, 111-123 (1989) MathReview

[H] Hirschhorn, P. S.: Model categories and their localizations. (Mathematical Surveys and Monographs, Vol. 99). Amer. Math. Soc. 2003 MathReview

[Kl] Klein, J. R.: Poincaré daulity embeddings and fiberwise homotopy theory. Topology 38, 597-620 (1999) MathReview

[K12] Klein, J. R.: Poincaré duality embeddings and fiberwise homotopy theory, II. Quart. Jour. Math. Oxford 53 319-335 (2002) MathReview

[K13] Klein, J. R.: Poincaré duality spaces. Surveys on surgery theory, Vol. 1, 135-165 Ann. of Math. Stud. 145, Princeton Univ. Press 2000 MathReview

[Kr] Kriz, I.: On the rational homotopy type of configuration spaces. Ann. of Math. 139, 227-237 (1994) MathReview

[L] Levitt, N.: Spaces of arcs and configuration spaces of manifolds. Topology 34, 217-230 (1995) MathReview

[L-S] Longoni, R., Salvatore, P.: Configuration spaces are not homotopy invariant. arXiv:math.AT/0401075

[St] Stallings, J. R.: Embedding homotopy types into manifolds. 1965 unpublished paper (see http://math.berkeley.edu/ stall for a TeXed version)

[Wa1] Wall, C. T. C.: Classification problems in differential topology-IV. Thickenings. Topology 5, 73-94 (1966) MathReview

[Wa2] Wall, C. T. C.: Surgery on Compact Manifolds. Second edition. Edited and with a foreword by A. A. Ranicki. Mathematical Surveys and Monographs 69 . Amer. Math. Soc. 1999 MathReview

[Wa3] Wall, C. T. C.: Poincaré complexes: I. Ann. Math. 86, 213-245 (1970) MathReview

Department of Mathematics, Wayne State University

Detroit, MI 48202, USA

Email: aouina@math.wayne.edu and klein@math.wayne.edu

Received: 29 January 2004 Revised: 4 July 2004 